\title{
A review of systemic therapy in biliary tract carcinoma
}

\author{
Holger Jansen ${ }^{1}$, Ulrich-Frank Pape ${ }^{2,3}$, Nalân Utku ${ }^{1,4}$ \\ ${ }^{1}$ Campus Virchow \& Mitte Charité, Institute f. Med. Immunologie, Berlin, Germany; ${ }^{2}$ Hepatology and Gastroenterology, Charité \\ Universitätsmedizin Berlin, Germany; ${ }^{3}$ Internal Medicine and Gastroenterology, Asklepios Klinik St. Georg, Asklepios Tumor Zentrum Hamburg, \\ Germany; ${ }^{4}$ CellAct Pharma GmbH, Dortmund, Germany \\ Contributions: (I) Conception and design: All authors; (II) Administrative support: All authors; (III) Provision of study materials or patients: All \\ authors; (IV) Collection and assembly of data: All authors; (V) Data analysis and interpretation: All authors; (VI) Manuscript writing: All authors; (VII) \\ Final approval of manuscript: All authors. \\ Correspondence to: Nalân Utku. Campus Virchow \& Mitte Charité, Institute f. Med. Immunologie, Berlin, Germany. Email: nalan.utku@charite.de.
}

\begin{abstract}
Biliary tract carcinoma (BTC) has a poor prognosis and is increasing in incidence. Although surgery, chemotherapy and other treatment modalities have improved, surgery remains the only potential curative treatment and is appropriate for only those few patients who present with localized, resectable disease. However, for the majority of patients, unresectable disease is evident at diagnosis and about $95 \%$ of patients die within 10 years, despite the majority receiving chemotherapy. Long-term survival is significantly greater for patients with resected BTC compared to those with unresectable disease. In unresected disease, life expectancy is limited, with first-line gemcitabine/cisplatin (GEM/CIS) accepted as standard of care. Currently no standard second-line regimen which provides significant improvement of clinical outcomes exists for those who present with refractory disease or who relapse after first-line treatment. Of particular importance is establishing the impact of best supportive care (BSC) as a benchmark for survival outcomes to which the impact of treatment modalities can be compared. Survival outcome often differs significantly for patients with different prognostic factor profiles even when receiving the same therapy so that it can be difficult to predict which patient subgroup might benefit most from which therapy. Therefore, the influence of prognostic factors on survival under different therapies as well as under BSC needs to be further assessed in order to arrive at truly evidence-based, best therapeutic decisions for individual patients. Encouraging new research into the genomic landscape of BTC may help to further subdivide the BTC population into molecular-genetic clusters likely to be sensitive to different targeted therapy approaches leading to further improvements in survival. Consequently, an unmet need exists not only to develop new and more effective therapies for this devastating disease, but also to integrate original research findings into a more complex, dynamic, individualized therapeutic decision model to aid clinicians in making evidence-based, best therapeutic decisions for individual patients.
\end{abstract}

Keywords: Biliary tract carcinoma (BTC); prognostic; unresectable; metastatic; chemotherapy

Submitted Apr 28, 2020. Accepted for publication Jul 13, 2020.

doi: 10.21037/jgo-20-203

View this article at: http://dx.doi.org/10.21037/jgo-20-203

\section{Introduction}

Biliary tract carcinoma (BTC), also termed cholangiocarcinoma (CCA), is a rare malignant disease comprising less than $1 \%$ of all cancers (1), but up to $30 \%$ of all primary liver tumors (2). Incidence is highest in SouthEast Asia (87.7/100,000) (2). Rates are low in Western countries at $0.2-3.5$ per $100,000(1,3,4)$. BTC incidence and mortality are increasing, making it one of the fastest rising cancers worldwide (4-7).

CCAs are classified as intrahepatic (iCCA), perihilar (pCCA), and distal (dCCA); the latter two are grouped as "extrahepatic" (eCCA), while pCCA is also known as "Klatskin Tumor" (4,5,8,9). Gallbladder carcinomas (GBCs) 
are distinct but often grouped with CCA (10).

While surgery is the only cure (11), about $60-85 \%$ of patients present with unresectable, or metastatic disease $(4,5)$; thus, prognosis is poor, with 5 -year survival rates of $4.1 \%$ for unresectable and $33.3 \%$ for resectable tumors (12). The final cure rate after surgery is $14.5 \%$ after 9.5 years $(13,14)$ equating to an overall cure rate of only about $4.1 \%$ of the initial diagnosed population after 10 years.

Treatment strategies, including systemic chemotherapy as the most important therapy backbone, have improved over time. However, due to disease rarity there is a lack of randomized controlled trials (RCTs) and studies comparing treatments to best supportive care (BSC), while most evidence originates from retrospective studies. Therefore, study outcomes vary greatly and are not directly comparable; this creates uncertainty among clinicians and researchers who should make evidence-based decisions in this heterogeneous disease area.

This article reviews current treatment practices, focusing on systemic therapy and associated outcomes in CCA at important clinical decision points, it summarizes key findings from the literature, and draws valuable conclusions for future research and clinical practice.

\section{Natural course of disease under BSC}

The clinical course of BTC varies depending on disease and patient condition as assessed by the Eastern Cooperative Oncology Group (ECOG) performance status (PS). In cases of metastatic BTC and good PS, patients are deemed "fit-for-chemotherapy"; for patients with metastatic disease and poor PS, BSC is usually preferred (15). BSC is also commonly used for management of refractory disease, relapse following first-line therapy, or recurrence after surgery; these all constitute different decision points along the collective patient treatment pathway with potentially different treatment outcomes, even under the same therapy (16).

The impact of BSC on overall survival (OS) following the natural disease course has not been widely studied; this is particularly true for the "fit-for-chemotherapy" population not having received chemotherapy (15). Understanding how outcomes are associated with prognostic factors throughout the natural disease course (i.e., without therapeutic interventions except BSC), may help to inform treatment decisions and improve patient survival. Key literature points for the natural disease course under BSC include.

\section{Historically, median OS under BSC was estimated to be about 3-6 months}

While studies with a BSC arm are rare, median overall survival $(\mathrm{mOS})$ in patients receiving BSC historically ranges from 2.5 to 5.7 months (CI: 0.2-9.6) (13,17-23). However, due to the mainly retrospective nature of studies and diversity of patients included, a selection bias may have assigned "older, sicker" patients who have a higher risk of premature death to BSC, which in turn leads to underestimation of survival, especially for patients deemed "fit-for-chemotherapy".

\section{Median OS under BSC in treatment-naïve patients is up to 7.1 months}

Only two retrospective studies have focused exclusively on natural disease outcome in treatment-naïve patients with BTC $(15,24)$. In the first study ( $\mathrm{n}=204$, PS $0-2)$, mOS was 7.1 months (range, $0.2-46.9$ months) (15); however, in the second study [ $\mathrm{n}=330, \mathrm{TNM}$ (Tumor, Node, Metastasis) stage I-IV patients], mOS was only 3.9 months (range, $0.2-67.1$ months) (24). This variation in mOS is best explained by the different study populations. In the first study, patients were exclusively "fit-for-chemotherapy" (PS $0-2$, with $60 \%$ of patients PS 0 or 1 , all anatomical subtypes); however, the second study included a sicker population (mainly stage III/IV patients, the majority iCCA cases, known to have shorter OS). These survival differences highlight the potential effect of selection bias and importance of comparing similar study populations when evaluating studies.

\section{Median OS under BSC is strongly prognostic factor-dependent}

Prognostic factors influence survival in patients receiving BSC. For example, in the first study above, mOS was shorter for women compared to men: 5.6 months (CI: 3.97.3 ) versus 8.3 months (CI: 6.8-9.9), $\mathrm{P}=0.315$ (15). Other factors statistically significantly influencing mOS included: locally advanced versus advanced disease (13.8 versus 6.2 months, $\mathrm{P}=0.001$ ), anatomic subtype [GBC 4.4 months, iCCA 4.7 months, eCCA 9.7 months, Ampulla of Vater (AoV) carcinoma 11.2 months, $\mathrm{P}=0.015]$, carcinoembryonic antigen (CEA) $(\geq 4 \mathrm{ng} / \mathrm{mL} 5.8$ months, $<4 \mathrm{ng} / \mathrm{mL}$ 10.6 months, $\mathrm{P}=0.006)$ and carbohydrate antigen $(\mathrm{CA}) 19-9$ $(\geq 100 \mathrm{U} / \mathrm{mL} 6.0$ months, $<100 \mathrm{U} / \mathrm{mL} 10.6$ months, 

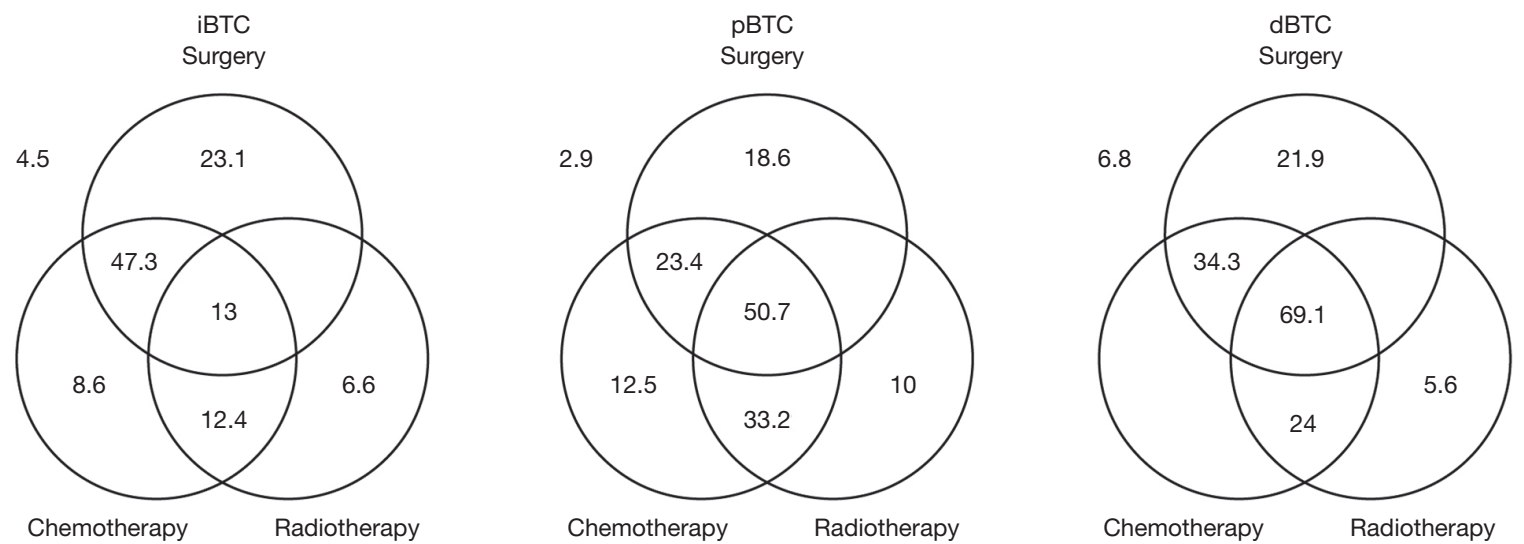

Figure 1 Median survival (months) for biliary tract carcinoma patients receiving single- or multi-modal treatment (32).

$\mathrm{P}=0.001)$ (15). In the retrospective study discussed above, survival differences were associated with anatomic subtype (iCCA versus pCCA), localized versus metastatic disease, albumin levels, TNM staging, CEA and bilirubin (24).

Importantly, surgery is not only a potential cure for BTC, but also a powerful prognostic factor leading to a median recurrence-free survival (mRFS) in patients receiving BSC of 18 months (CI: 13-28) (25) and 22 months (CI: 13.6-38.3) (26), with an additional mOS of 8.0 months for patients receiving BSC after recurrence (27). This adds up to a total mOS of 26-30 months for resected patients receiving BSC compared to 3-7 months for unresected treatment-naïve patients.

\section{In rare cases, survival in patients receiving BSC is up to 5.5 years}

Rare BTC cases have survived up to 37.7 months (24), 46.9 months (15) and 67.1 months (24) i.e., 3-5.5 years whilst receiving BSC only. While patients demonstrating such relatively long survival exist in almost every trial, the reasons for these long survival times, in the absence of therapies, remain unclear and warrant further investigation.

\section{Treatment outcome for patients with resectable BTC}

Surgery is the only potential cure for TNM R0 resected patients without lymph node metastases (11); however, even with surgery, life expectancy remains limited. While an extensive liver resection is almost always required to achieve R0 (28), liver transplantation is only performed in 2.2\% (29) of patients. Therefore, this review focuses only on outcomes following standard resection surgery. Key literature points for surgical treatment are:

\section{The majority of newly diagnosed CCA cases remain unresectable}

Only $15-40 \%$ of newly diagnosed patients progress to surgery $(4,5,30,31)$, preferably performed at expert centers, while $60-85 \%$ of patients remain unresectable.

\section{Survival for resected patients is significantly longer compared to unresected patients}

A retrospective study $(\mathrm{n}=242)$ showed resected patients have better OS than unresected patients independent of additional treatments (Figure 1) (32). Another study reported significantly longer mOS in resected compared with unresected patients (37 versus 14 months, $\mathrm{P}<0.001$ ) (31), while a third study showed superior mOS in patients who underwent surgery with curative intent (26.3 months) when compared with patients who underwent palliative surgery (7.3 months; $\mathrm{P}<0.001)$ or no surgery (2.6 months, $\mathrm{P}<0.001)$ (33). Therefore, unsurprisingly, resected patients survive significantly longer than unresected patients.

\section{Surgically pre-treated patients have a longer survival compared to unresected patients even when receiving the same therapy}

In BT-22, mOS for resected patients was compared to unresected patients when receiving the same treatment [gemcitabine/cisplatin (GEM/CIS): 16.1 versus 9.4 months, GEM: 12.7 versus 7.4 months] (34). Two recent large 
Table 1 Overall survival rates for all anatomical biliary tract carcinoma subtypes after resection (37)

\begin{tabular}{lcccc}
\hline OS (year) & All BTC subtypes $(\%)$ & iBTC (\%) & pBTC (\%) & dBTC $(\%)$ \\
\hline OS at 1 year & 78 & 85 & 70 & 43 \\
OS at 3 years & 49 & 52 & 45 & 47 \\
OS at 5 years & 31 & 34 & 28 & 23 \\
\hline
\end{tabular}

dBTC, distal biliary tract carcinoma; iBTC, intrahepatic biliary tract carcinoma; OS, overall survival; pBTC, perihilar biliary tract carcinoma.

RCTs [BILCAP $(25,35)$ and PRODIGE-12 (26)], which investigated the effects of adjuvant chemotherapy, also reported longer survival times for resected patients compared with unresected patients who were receiving the same treatment [BILCAP: capecitabine mOS 51 months, BSC 36 months; PRODIGE-12: mRFS of 30.4 months for gemcitabine/oxaliplatin (GEMOX), BSC 22 months], compared to mOS of 11.7 months for GEM/CIS (36) and 7.1 months for BSC (15) in unresected patients. Other studies also support these findings, with surgery improving outcomes in both early and advanced stages of disease (32). Thus, as could be anticipated, resected patients have longer survival than unresected patients when treated with the same therapy. In order to allow a full and fair assessment of different treatment regimens, studies should ideally not enroll a mixed population of resected and unresected patients.

\section{After adjustment for baseline characteristics, there is no difference in long-term survival after surgery between BTC anatomical subtypes}

Studies have revealed that after adjusting for baseline characteristics, there are no significant differences in postsurgical long-term survival between different anatomical subtypes of $\mathrm{BTC}(\mathrm{P}=0.127$, Table 1) $(32,37)$.

\section{Surgical and other treatment outcomes have improved over time}

In a retrospective study, 144 iCCA patients were divided into two groups: Period 1 [1993-2006] prior to new therapies (adjuvant chemotherapy, multimodal therapy after recurrence), and Period 2 [2006-2014] after the arrival of new therapies (38). Survival improvements were evident from Period 1 to 2 with mOS increasing from 21.4 to 57.7 months $(\mathrm{P}<0.001)$, median disease-free survival (mDFS) from 12.2 to 16.6 months $(\mathrm{P}=0.027)$, survival after recurrence from 8.0 to 22.3 months $(\mathrm{P}<0.001)$, and survival with Node-positive (N1) status from 12.4 to 26.0 months $(\mathrm{P}=0.0012)$. The advent of structured GBC staging was also associated with improved survival (39).

\section{Even with curative intent surgery, the actual overall cure rate remains very low at $4.1 \%$}

Using a "cure-fraction-model", Spolverato et al. showed a surgical cure probability of only $14.5 \%$ (CI: 8.7-23.2) with a time to cure of 9.5 years; mOS for not cured patients was 21.6 months (1.8 years) (40). Therefore, even with surgery, $50 \%$ of patients die within 2 years and $71 \%$ of the remaining $50 \%$ of patients die within the next 8 years. Patients who survive the first 2 years post-surgery, therefore, still have only a $29 \%$ chance of cure. Assuming a surgical exploration rate of $45 \%$, of which $63 \%$ of surgeries are resections (31), leads to an estimated overall cure rate of only $4.1 \%(45 / 100 \times 63 \times 14.5 / 100=4.1 \%)$; thereby, $95.9 \%$ of patients die within 10 years.

\section{Survival outcomes after surgery differ significantly in the literature}

There is a confusingly wide range of post-surgical survival estimates in the literature. Overall, 5-year survival rates range from $13-63 \%$ and mOS from 9-69 months ( $<1-5.75$ years) (Table 2). Improved survival over time and differences in prognostic factor distribution of study populations may explain this variability. However, contemporary mOS may range from 36.4 months (in patients receiving BSC) to 51.1 months (for patients treated with capecitabine), as observed in the BILCAP study $(25,35)$.

\section{Survival after surgery varies depending on prognostic factor profile}

While surgery strongly influences the outcome of subsequent treatment, variations in survival post- 
Table 2 Studies providing survival estimates for cholangiocarcinoma patients after surgery

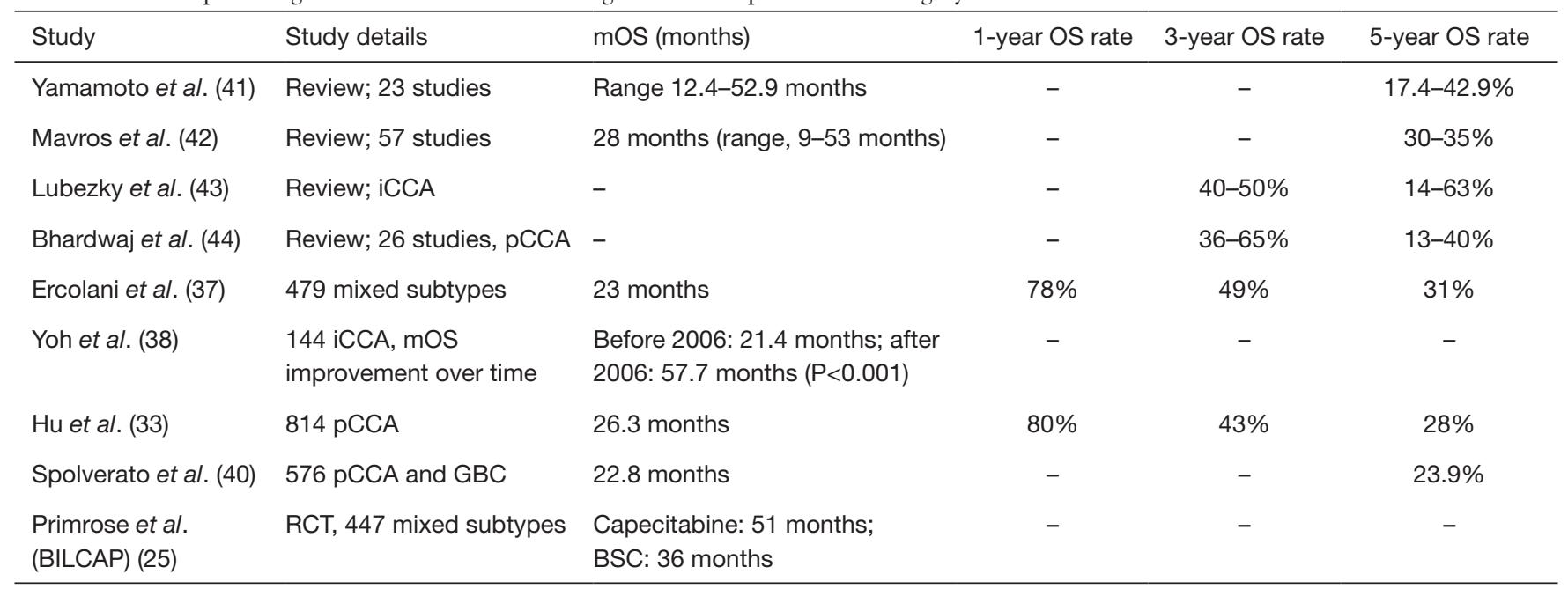

BSC, best supportive care; dCCA, distal cholangiocarcinoma; GBC, gall bladder carcinoma; iCCA, intrahepatic cholangiocarcinoma; m, months; mOS, median overall survival; OS, overall survival; pCCA, perihilar cholangiocarcinoma; RCT, randomized controlled trial.

surgery have been observed. In a retrospective study in pCCA patients $(n=814)$, surgical margin, lymph node metastases, tumor differentiation and vascular invasion were independent prognostic factors for OS (33). In these patients, variation in baseline prognostic factors altered median OS (26.3 months) from 12.4 months $(-52.9 \%)$ to 54.1 months $(+105.7 \%)$ (Table 3) (33), and these changes may have been independent of treatment. In another metaanalysis (23 studies, 2,063 dCCA patients) prognostic factor-dependent 5 -year survival ranged from $15.1 \%$ to $65.7 \%$. Negative resection margin, absence of lymph node metastases and absence of perineural invasion were associated with longer survival (Table 4) (45).

Independent prognostic factors for better outcome, including no lymph node metastasis and CA-19-9 $<50 \mathrm{U} / \mathrm{mL}$, were also identified for 576 patients with eCCA (40). Patients with CA-19-9 $<50 \mathrm{U} / \mathrm{mL}$ and no lymph node metastases had a shorter modelled cure fraction of $39 \%$ after 4.1 years compared to $5.1 \%$ after 6.8 years for patients with a CA-19-9 level $>50 \mathrm{U} / \mathrm{mL}$ and lymph node metastases (40). Furthermore, post-surgical OS is influenced by TNM stage at diagnosis (32): stage I (23 months), II (25 months), III (14 months), IV (4.5 months). Survival after surgery therefore varies widely between prognostic factor subgroups and patients benefit differently from surgery depending on their individual prognostic factor profile.

\section{Adjuvant chemotherapy and radiotherapy after surgery}

The rationale for adjuvant therapy is high recurrence and poor survival after surgery (46), despite safety concerns after hepatectomy, as standard doses of chemotherapy are not well tolerated; conversely dose reduction limits efficacy (47). Key literature findings for adjuvant therapy are:

\section{Adjuvant therapy is controversial and prognostic factor dependent}

Adjuvant therapy is controversial (48). A systematic review and meta-analysis (20 studies, $\mathrm{n}=6,712$ ) showed a trend towards statistically non-significant improvement in OS with any adjuvant therapy compared to surgery alone [pooled odds ratio (OR) 0.74, $\mathrm{P}=0.06$ ] (49). However, benefit was significantly greater for chemotherapy alone $(\mathrm{OR}=0.39, \mathrm{P}<0.001)$ and chemoradiotherapy $(\mathrm{OR}=0.61$, $\mathrm{P}=0.049)$ versus radiotherapy alone $(\mathrm{OR}=0.98, \mathrm{P}=0.90)$. Node positive $(\mathrm{N} 1)(\mathrm{OR}=0.49, \mathrm{P}=0.004)$ or resection margin positive $(\mathrm{R} 1)(\mathrm{OR}=0.36, \mathrm{P}=0.002)$ high-risk patients demonstrated a significantly greater benefit following adjuvant therapy than $\mathrm{R} 0$ patients $(\mathrm{OR}=1.26, \mathrm{P}=0.20)$ (49). Thus adjuvant therapy is often recommended in patients with a less favorable prognostic factor profile $(48,49)$.

In contrast, another systematic review (57 studies, $\mathrm{n}=4,756$ ) in iCCA patients, showed adjuvant chemotherapy or radiotherapy as not having a beneficial effect, with vascular invasion and lymph node metastasis associated with 
Table 3 Prognostic factors for OS after surgery in hilar biliary tract carcinoma patients (33)

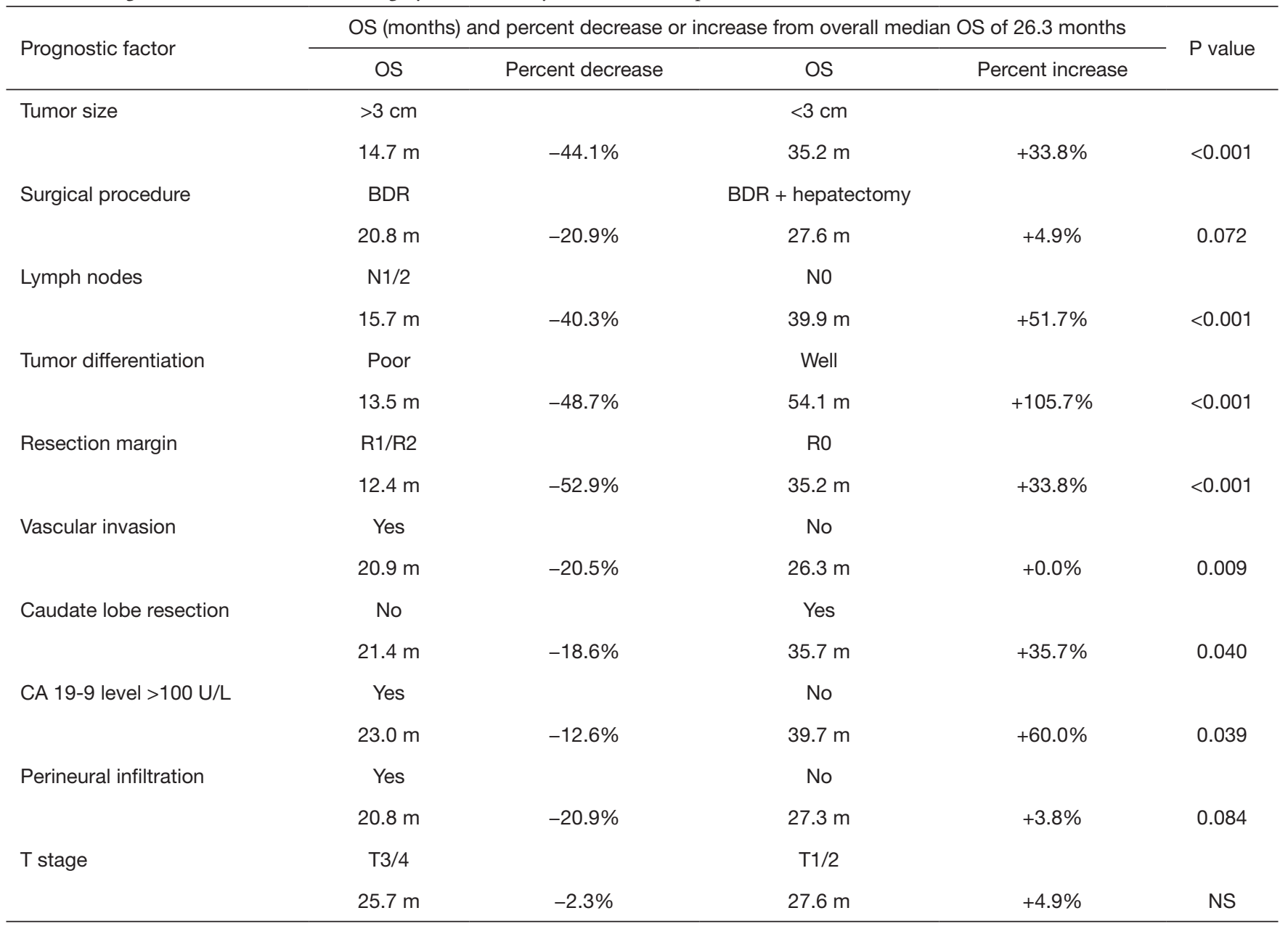

BDR, hilar bile duct resection; CA 19-9, carbohydrate antigen 19-9; OS, overall survival.

Table 4 Influence of prognostic factors on 5 -year survival after surgery in distal biliary tract carcinoma patients (45)

\begin{tabular}{|c|c|c|c|c|c|}
\hline Factor & \multicolumn{2}{|c|}{ 5-year survival } & Relative risk (RR) & $95 \% \mathrm{Cl}$ & $P$ value \\
\hline \multicolumn{6}{|c|}{ Factors without influence on survival } \\
\hline Gender & Male, $38.8 \%$ & Female, $35.0 \%$ & 0.95 & $0.68-1.32$ & 0.76 \\
\hline Age, years & $<65,35.6 \%$ & $>65,34.4 \%$ & 1.31 & $0.82-2.12$ & 0.26 \\
\hline \multicolumn{6}{|l|}{ Factors with influence on survival } \\
\hline Perineural invasion & $31.3 \%$ & $65.7 \%$ & 0.51 & $0.40-0.64$ & $<0.00001$ \\
\hline Lymph node metastases & $23.7 \%$ & $47.2 \%$ & 0.51 & $0.38-0.70$ & $<0.0001$ \\
\hline Negative resection margins & $40.8 \%$ & $15.1 \%$ & 2.11 & $1.36-3.3$ & 0.001 \\
\hline
\end{tabular}

$\mathrm{Cl}$, confidence interval; diff, differentiated; $\mathrm{RR}$, relative risk. 
Table 5 Overview of studies providing median recurrence-free survival (mRFS) time and recurrence rates for biliary tract carcinoma after surgery

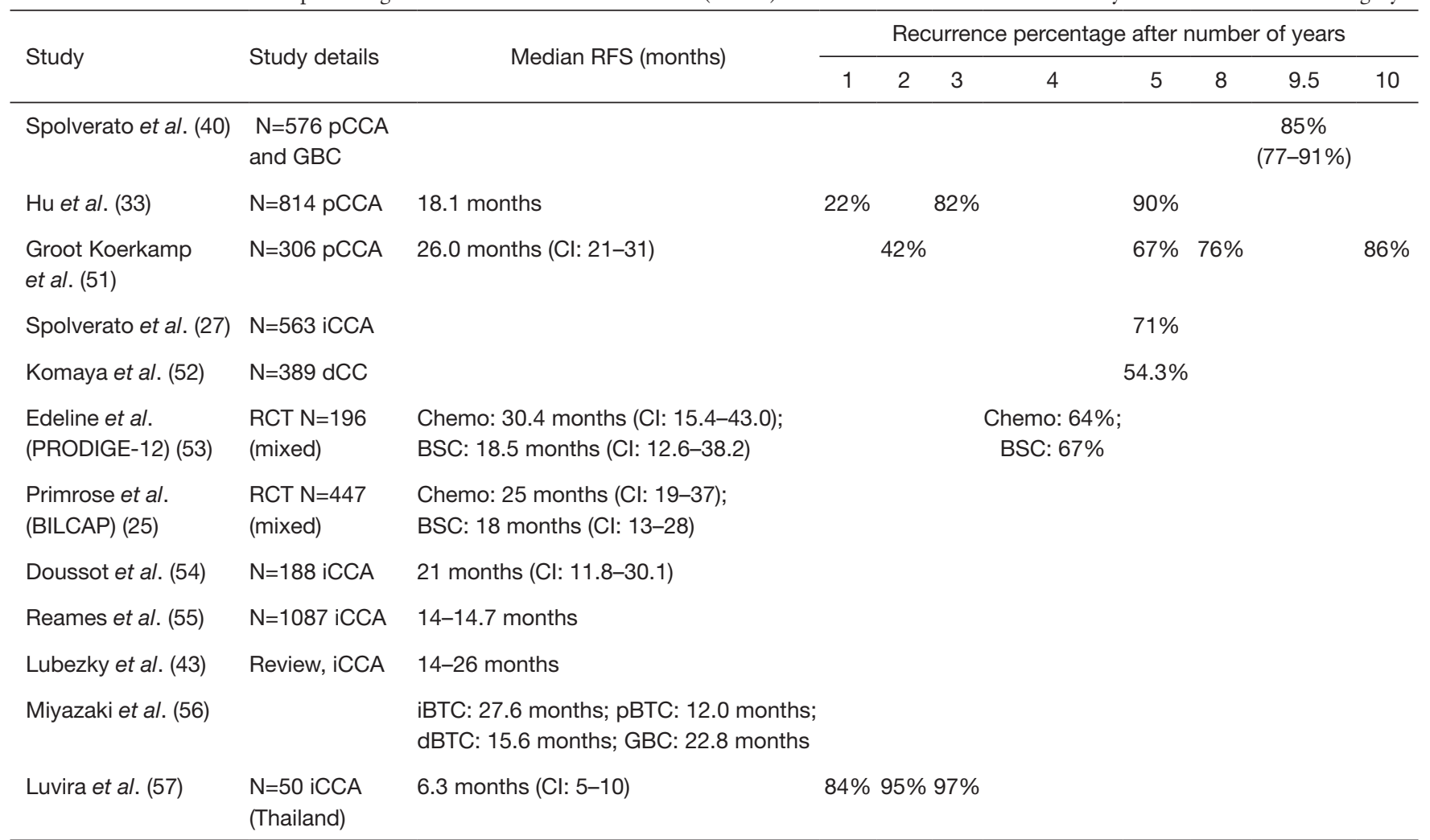

BSC, best supportive care; Cl, confidence interval; dBTC, distal biliary tract carcinoma, gall bladder cancer; iBTC, intrahepatic biliary tract carcinoma; m, months; pBTC, perihilar biliary tract carcinoma; RFS, recurrence-free survival; RCT, randomized controlled trial.

shorter OS (42). Other retrospective studies also present conflicting viewpoints, with some demonstrating $(14,47,50)$ and others not showing a benefit with the use of adjuvant therapy compared with BSC $(40,45)$.

\section{Results of RCTs (PRODIGE-12, BILCAP) continue the controversy of adjuvant therapy}

PRODIGE-12 reported no statistically significant difference in mRFS for GEMOX (30.4 months, CI: 15.4-43.0) compared to BSC (18.5 months, CI: 12.6-38.2) (26). Moreover, BILCAP (ITT population) showed a statistically non-significant survival trend in mOS for capecitabine (51.1 months, CI: 34.6-59.1) over BSC (36.4 months, CI: 29.7-44.5) (35). However, sensitivity analyses for BILCAP's per-protocol population, adjusting for gender, disease grade, and nodal status demonstrated a statistically significant OS advantage for capecitabine (HR $=0.71$, CI: $0.55-0.92$ ), and a statistically significant difference in $\mathrm{mOS}$ for capecitabine (53 months) compared with BSC (36 months; HR $=0.75$, CI: 0.58-0.97, $\mathrm{P}=0.028)$ (25). Nevertheless, capecitabine was not associated with a statistically significant increase in mRFS (24.4 months, CI: 18.6-35.9) versus BSC (17.5 months, CI: 12.0-23.8) (35). While the BILCAP authors concluded that "Capecitabine can improve OS in BTC when used as adjuvant chemotherapy following surgery and should be considered as standard of care" (35), the majority of data derived from RCTs suggest an overall non-statistically significant trend in favour of adjuvant chemotherapy; it is therefore possible that this treatment approach may benefit only certain patient subgroups.

\section{Recurrence-free survival (RFS) and disease-free survival (DFS) after resection}

\section{Median RFS is 12-30 months with highest recurrence risk within the first 2 years}

Median RFS after resection is $12-30$ months (Table 5). Most recurrences happen within 5 years, the first 2 years conferring highest risk (Figure 2); mRFS was independent of BTC location (27). 


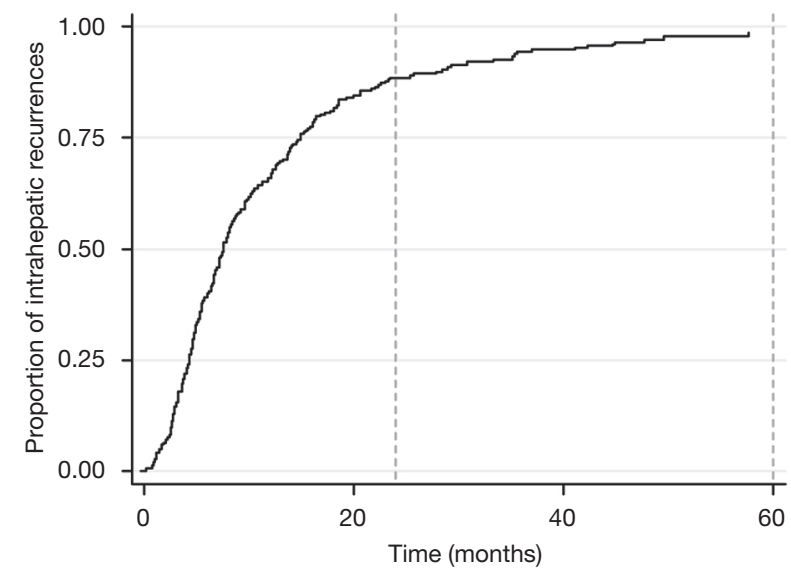

Figure 2 Overall risk of intrahepatic biliary tract carcinoma recurrences at 2 and 5 years (27).

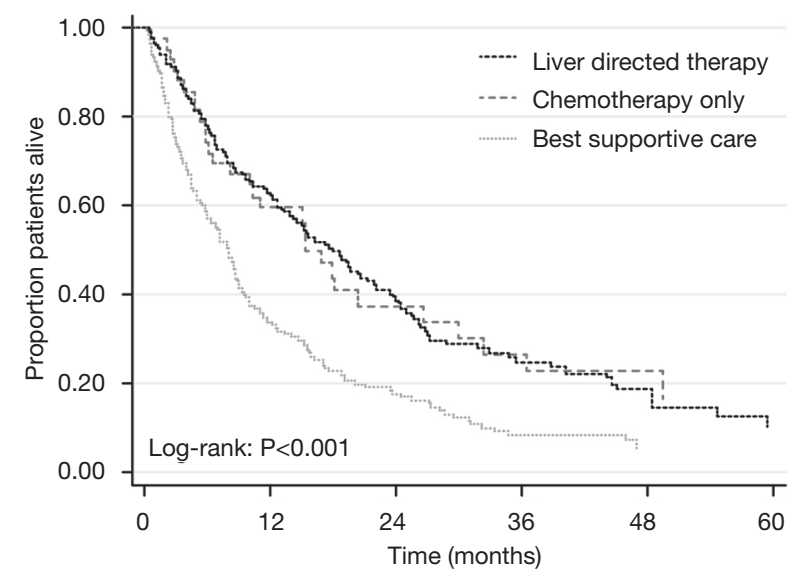

Figure 3 Five-year overall survival after treatment of biliary tract carcinoma recurrence with either liver-directed therapy, chemotherapy, or best supportive care (27).

RFS and DFS improve over time and are prognostic factor dependent

A study in iCCA patients (1993-2006, $\mathrm{n}=65 ;$ 2007-2014, $\mathrm{n}=79$ ), observed a marked improvement in post-surgical mDFS post-2006 (16.6 months) with the introduction of adjuvant chemotherapy versus pre-2006 (12.2 months; $\mathrm{P}=0.027$ ) (38). Furthermore, another retrospective study in 389 dCCA patients identified the following independent prognostic factors for time to recurrence and RFS: perineural invasion $(\mathrm{P}=0.001$ and $\mathrm{P}=0.009)$, pancreatic invasion (both $\mathrm{P}<0.001$ ) and lymph node metastases (both $\mathrm{P}<0.001)$. Rates for 5 -year RFS worsened with an increasing number of risk factors $(70.6 \%, 50.3 \%, 31.8 \%$ and $13.4 \%$ for
0, 1, 2, 3 factors present, respectively) (52); thus, it would appear that prognostic factor combinations have an additive effect and RFS is dependent upon risk factor profile. Several models to estimate patient prognosis exist (54).

\section{Treatment of post-surgical recurrence}

The majority of relapse patients receive liver-directed therapy including surgery

In a study of 563 iCCA patients with post-surgical recurrence, $47.5 \%$ received therapy and $52.5 \%$ BSC; of those with therapy, the majority (75.8\%) received repeat liver-directed therapy (intra-arterial therapy, repeat resection, ablation) \pm chemotherapy, while only $24.2 \%$ received chemotherapy alone (27).

\section{Outcomes with liver-directed therapy may be better} than chemotherapy alone or BSC

A study of 563 iCCA patients with post-surgical relapse demonstrated that the mOS from time of recurrence was 11.1 months (BSC 8.0 months, chemotherapy 16.8 months, liver-directed-therapy 18 months, $\mathrm{P}<0.001$; Figure 3) (27). In another retrospective study, 107 recurrent patients received either surgery $(n=14)$, chemotherapy $(n=45)$, or BSC $(n=48)(56)$. Five-year survival was significantly better after surgery versus chemotherapy or BSC: $19 \%$ versus $5.3 \%$ versus $0 \%$, respectively $(\mathrm{P}<0.0001)$. Factors influencing outcomes included: residual primary tumor status (HR $=1.58$, CI: $1.00-2.44, \mathrm{P}=0.047)$, time to recurrence $\geq 1$ year (HR $=0.62$, CI: $0.39-0.97, \mathrm{P}=0.037)$, and surgery for recurrence $(\mathrm{HR}=0.32, \mathrm{CI}: 0.14-0.62, \mathrm{P}<0.001$ ). Furthermore, in 220 patients undergoing surgery for recurrent BTC (systematic review, 10 studies), median mOS was 26.1 months (range, 10.0-66.6 months), with 3-year and 5 -year survival rates after recurrence of $51.4 \%$ (range, $29-100 \%$ ) and $29 \%$ (range, 0-51.4\%), respectively (56). Therefore, repeat therapy including surgery may offer a better choice for recurrent BTC than chemotherapy alone.

\section{First-line systemic chemotherapy in mainly unresected patients}

\section{Gemcitabine plus cisplatin (GEM/CIS) is the accepted standard for first-line therapy}

In the absence of an established first-line standard of care treatment, due to a lack of evidence from RCTs, Eckel et al. published a pooled analysis of 161 published trials (58). 
The findings from this pooled analysis demonstrated that the best outcomes were achieved in those patients treated with a GEM/platinum combination, resulting in the recommendation of triple-agent chemotherapy consisting of a GEM/platinum/fluoropyrimidine combination as provisional first-line standard of care therapy. In 2010, the ABC-02 trial (36) established the superiority of GEM/ CIS over GEM alone $(n=410)$ resulting in the recognition of GEM/CIS as standard of care first-line therapy for locally advanced unresectable and metastatic BTC (59). For GEM/CIS and GEM, respectively, mOS was 11.7 months (CI: 9.5-14.3) versus 8.1 months (CI: 7.1-8.7), median progression-free survival (PFS) was 8.0 months (CI 6.6-8.6) versus 5.0 months (CI: 4.0-5.9), and disease control rate (DCR) was $81.4 \%$ versus $71.8 \%$ (36). Findings from the BT-22 study ( $n=84)$ supported the use of GEM/ CIS combination therapy (34). For GEM/CIS and GEM, respectively, mOS was 11.2 months (CI: 9.1-12.5) versus 7.7 months (CI: 6.1-11.0), median PFS was 5.8 months (CI: 4.1-8.2) versus 3.7 months (CI: 2.1-5.3), and DCR was $68.3 \%$ versus $50.0 \%$ (34). A meta-analysis of both studies confirmed the superiority of GEM/CIS over GEM and concluded that GEM/CIS reduced death/progression risk by $35 \%$ compared with GEM (60). These findings were further supported by RCTs, retrospective studies and prospective observational studies $(61,62)$; importantly, however, none of these studies included BSC as a reference point.

GEMOX was also identified as a first-line standard of care, with better outcomes (mOS 9.5 months; mPFS 8.5 months) versus 5 -fluorouracil/folinic acid (5-FU/FA) (mOS 4.6 months; mPFS 3.5 months) and BSC (mOS 4.5 months; mPFS 2.8 months) (18). However, a systematic review $(n=1,470)$, found a weighted median of mOS of 9.7 months for GEM/CIS and 9.5 months for GEMOX, with GEM/CIS having greater toxicity (63). Sensitivity analysis of the six studies using the ABC-02 CIS dose revealed small improvement in mOS to 11.7 months, with an increased toxicity (63). Therefore, GEM/CIS (with standard CIS) may exhibit an OS advantage of 2 months but at the cost of higher toxicity (63).

\section{Variable outcomes with GEM/CIS treatment have been reported}

GEM/CIS outcome varies with some more recent studies, including large sample size reviews, supporting a shorter mOS of about 9.5 months (range, 8.4-10.5 months) (Table 6)
(59,63,65-69), versus 11.7 months (CI: 9.5-14.3) in ABC-02 (36). Eckel et al. were the first to suggest the use of GEM/CIS as standard of care in 2007 (58), deriving a mOS of 9.5 months for GEM/CIS in their second pooled analysis conducted in 2014 (69); this currently remains the largest available study.

\section{The treatment advantage gap for GEM/CIS over BSC in all patients is converging}

A 2010 review of BTC treatment suggested that no survival benefit for chemotherapy compared with BSC alone had been shown (70). Results from the randomized Phase III ABC-02 study demonstrated a survival advantage of cisplatin and gemcitabine doublet-chemotherapy over gemcitabine monotherapy; GEM/CIS thus became accepted as first-line standard of care therapy (36). Whilst there have been suggestions that the outcomes for GEM/CIS observed in ABC-02 may represent the top end of the range, with real-world mOS likely to be around 9.5 months (range, 8.4-10.5 months) and mPFS about 5-6 months (Table 6), outcomes for BSC have historically been underestimated with more recent data showing mOS with BSC of 7.1 months for "fit-for-chemotherapy" patients (15), and around 26-36 months for resected patients $(15,25,27)$. As previously discussed, differences in survival between adjuvant chemotherapy and BSC observed in the PRODIGE-12 (26) study were not statistically significant. The latest published findings from the second-line ABC06 study (23) further support this, with mOS in those from the control arm who received active symptom control (ASC) noted as 5.3 months compared to 6.2 months with chemotherapy.

Therefore, in the absence of RCTs directly comparing first-line GEM/CIS to BSC in a purely unresected, "fitfor-chemotherapy" population, it is possible to hypothesize that, at least for certain patient subgroups, there may be very little, if any, benefit when treating with GEM/ CIS compared to BSC. Given the potential impact of chemotherapy on patient quality of life, the question of clinical relevance of such a blanket treatment approach seems warranted in this era of personalized medicine.

\section{Best therapy decisions strongly depend on an individual's clinical profile}

Evidence suggests that GEM/CIS may be more effective in certain BTC subgroups. For example, in treatment-naïve 


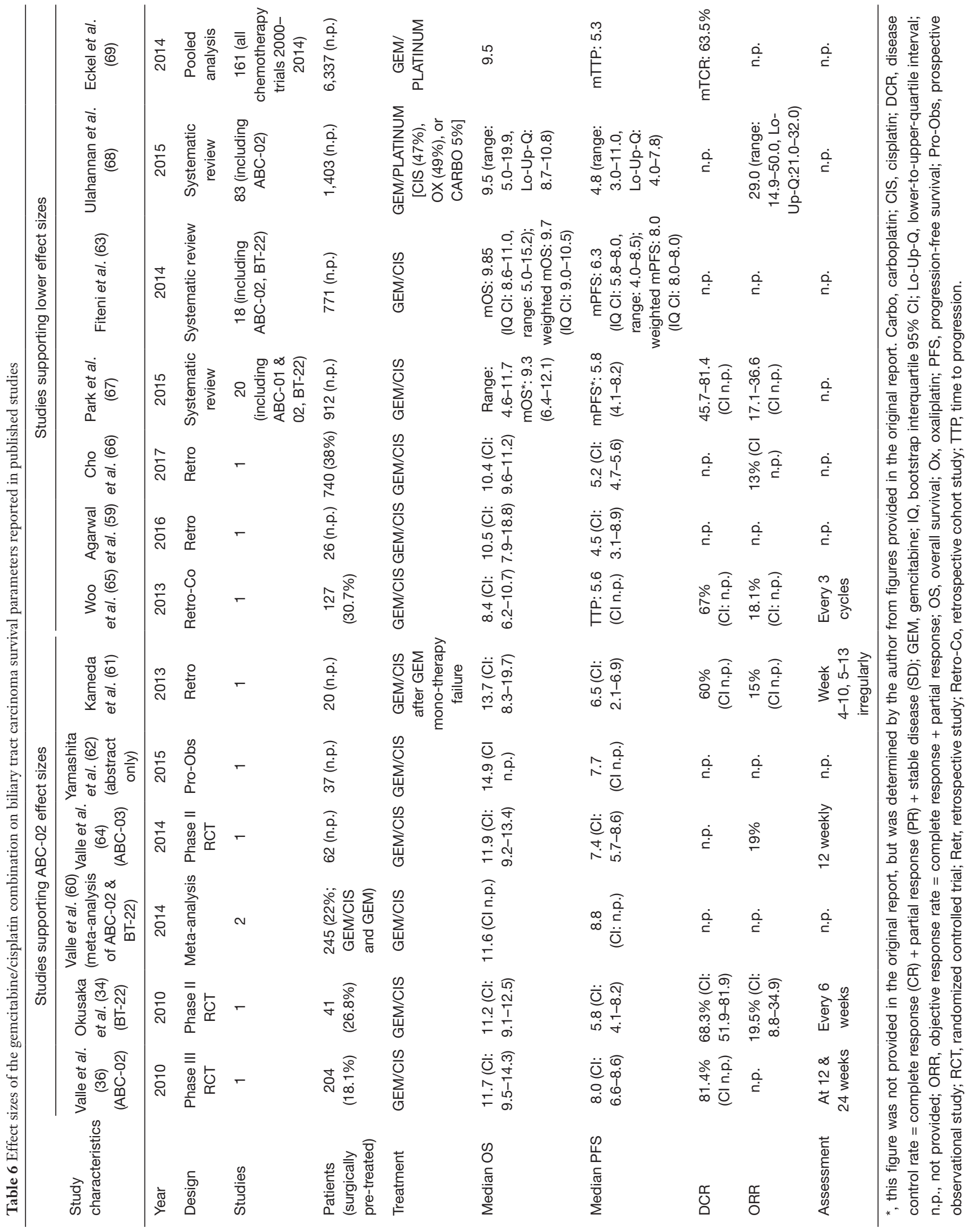


patients with metastatic BTC, the overall mOS for those receiving BSC was 7.1 months; however, for GBC mOS was only 4.4 months (CI: 2.9-5.9) (15). In contrast, mOS for GBC under GEM/CIS in BT-22 was 9.1 months (CI: 6.9-11.6) versus an overall mOS of 11.2 months (CI: 9.1-12.5) (34). A systematic review of data from studies examining GEM/CIS in the treatment of BTC confirmed these findings and suggested that GEM/CIS may be effective in GBC compared to BSC $(11,63,68)$.

Furthermore, according to a recent analysis pooling all data for iCCA from the ABC trials, the mPFS and mOS of iCCA in patients treated with GEM/CIS were 8.4 months (CI: 5.9-8.9) and 15.4 months (CI: 11.1-17.9) respectively (71). Therefore, a superior outcome in terms of OS was observed in patients with iCCA treated with GEM/CIS compared with the outcomes observed in patients with subtypes of eCCA, findings that should be considered for future clinical trial design. However, it should be noted that a short mOS has previously been observed in iCCA patients receiving BSC (4.7 months, CI: 3.54-5.87) (15), suggesting that GEM/CIS should remain the first-line treatment choice for patients with iCCA.

However, for patients who have extrahepatic CCA subtypes, constituting the majority of cases (60-95\%) $(5,8-10)$, the advantage of GEM/CIS over BSC is less clear. Under BSC, mOS for eCCA was 9.7 months (CI: 6.4912.91) and for AoV CCA 11.2 months (CI: 5.1-17.3) (15). These BSC survival data are similar to the corrected mOS of 9.5 months (range, 8.4-10.5 months) for GEM/ CIS (Table 6). Furthermore, for eCCA and AoV, a clear survival benefit for standard GEM/CIS over BSC cannot be confidently assumed, unless additional factors further influence outcomes. Therefore, chemotherapy-containing regimens are not the only option for consideration. Instead, factors such as locally advanced versus metastatic disease, luminal versus liver metastases, bilirubin $\geq 1.5 \times \mathrm{ULN}$ (upper limit of normal), CEA $>4 \mathrm{ng} / \mathrm{mL}$, CA-19-9 (elevated versus normal), ECOG PS (2 versus 0-1), RECIST criteria (versus non-measurable), previous surgery and associated factors, molecular genetic profile and other factors depending on the specific decision point along the collective patient treatment pathway all modify outcomes and should be considered in treatment decisions $(15,16,34,66)$.

For BSC versus GEM/CIS, prognostic factors have the potential to increase or decrease survival significantly $(15,16,34,66)$, and it is possible that the effect of multiple factors may be additive $(52,59)$. This impact on survival was evident in a small retrospective study of 26 patients receiving GEM/CIS (59). The presence of three risk factors (PS $\geq 2$, CEA $>3 \mathrm{ng} / \mathrm{mL}$, Stage $\mathrm{IVb}$ ) resulted in a $72 \%$ decrease in mOS from 10.5 to 2.9 months; conversely, a $71 \%$ increase in mOS from 10.5 to 18 months was observed in the absence of all three prognostic factors (59). Therefore, for a patient in whom the presence of all three factors has been identified, the value of additional treatment with chemotherapy, even in those considered to still be "fitfor-chemotherapy", should be queried.

Overall, this leads to a complex evidence-based therapeutic decision model for CCA, where static average survival figures for different therapy options are no longer the only consideration, but the influence of prognostic factors on outcome should also be considered in order to arrive at truly evidence-based therapeutic decisions for individual patients.

\section{GEM/CIS is effective in patients who present with signs of biliary tract obstruction due to luminal disease despite optimal stenting}

Another consideration is biliary tract obstruction, present in $70-84 \%$ of patients (16), and associated complications. The ABC trials excluded such patients; however, a benefit of chemotherapy has been shown in this patient group (16). Bilirubin normalized in $64 \%$ of patients during/after chemotherapy, with toxicity/outcomes comparable to $\mathrm{ABC}$ 02 [mPFS: 6.9 months (CI: 4.4-9.0); mOS 9.5 months (CI: 5.7-12.8)] (16,36). Patients whose bilirubin normalized during/after chemotherapy had a significantly higher DCR compared to patients whose levels did not (86\% versus $30 \%, \mathrm{P}=0.004)(16)$. While baseline bilirubin had no impact on PFS or OS, bilirubin normalization during/after chemotherapy was related to longer mOS compared to levels that did not normalize (11.4 versus 2.9 months, $\mathrm{HR}=0.49$, CI: $0.2-1.1, \mathrm{P}=0.08)$ (16). Moreover, patients with luminal disease-related obstruction (76\%) had better outcomes than patients with liver metastases (24\%): mPFS 7.0 months versus 2.6 months $(\mathrm{P}=0.1633)$, mOS 9.8 months versus 4.4 months $(\mathrm{HR}=0.74, \mathrm{P}=0.465)$ (16). These data suggest that GEM/CIS is an appropriate treatment approach for patients with advanced BTC (PS 0-1) and high bilirubin due to luminal disease despite optimal stenting (16).

\section{Other potentially more effective combinations than GEM/ CIS have been identified}

Potentially more effective combination chemotherapies have 
been identified $(68,69)$; these include GEM/platinum/5FU (mOS 12.5 months) or a GEM/epidermal growth factor receptor (EGFR) targeted therapy combination (mOS 12.7 months), which have been suggested as new standards for patients with good PS (69). Better outcomes for GEM/5-FU versus GEM/platinum were also demonstrated: mOS 12.5 versus 9.5 months, $\mathrm{P}=0.047(68,69)$.

\section{Second-line chemotherapy}

Outcome of second-line chemotherapy is poor-mPFS 2-3 months, mOS 6-7 months

No standard second-line therapy for BTC currently exists, and no clear advantage of one regimen over another has yet been established (72-74). In a retrospective study $(n=603), 196$ patients $(32.5 \%)$ received second-line chemotherapy, of which $43 \%$ (14\% of original cohort) also received third-line chemotherapy (72). The most common second-line regimens used were FOLFIRI (folinic acid, fluorouracil and irinotecan) and XELIRI (irinotecan and capecitabine) ( $\mathrm{n}=64)$, followed by 5 -FU or capecitabine $(\mathrm{n}=40)$, 5-FU plus cisplatin ( $\mathrm{n}=38$ ), and FOLFOX (folinic acid, fluorouracil and oxaliplatin) or XELOX (oxaliplatin and capecitabine; $\mathrm{n}=21$ ) (72). mPFS for second-line therapy was 3.2 months (CI: 2.8-4.0) and mOS 6.7 months (CI: 5.6-7.8), with no significant difference between regimens (72). Two retrospective studies of capecitabine + cisplatin demonstrated similar outcomes: $n=40$, mPFS 2.3 months, mOS 6.3 months (75); and $\mathrm{n}=294$, mPFS 2.8 months, mOS 7.7 months (76). In addition, a recent prospective Phase III trial of capecitabine + oxaliplatin $(\mathrm{n}=108)$ noted mPFS 5.3 months, mOS 10.6 months (77). Furthermore, a systematic review of 20 studies produced a weighted mOS of 7.2 months (CI: 6.2-8.2) (78) and a meta-analysis of 23 prospective Phase II trials plus 9 retrospective studies, involving a total of 1,391 patients, demonstrated a weighted mPFS of 2.6 months and weighted mOS of 6.5 months (79). Treatment using a platinum + gemcitabine combination has also been shown to be superior to monotherapy (mOS 7.1 versus 5.0 months, $\mathrm{P}=0.006$ ) (73). Overall, however, patients demonstrate poor outcomes with no clear advantage shown for one therapeutic regimen over another.

Prognostic factors for better outcomes following second-line therapy included CA-19-9 ( $<157$ versus $\geq 157 \mathrm{U} / \mathrm{mL}$, and $\leq 400$ versus $>400 \mathrm{U} / \mathrm{mL})$, ECOG PS ( 0 versus $1-2 / 2-3)$, bilirubin $(\leq 17$ versus $>17 \mu \mathrm{mol} / \mathrm{L}$ ), absence of distant metastases, and disease control during first-line therapy $(72,73)$.

\section{Recent developments in second-line chemotherapy}

Prolongation of first-line therapy may prolong survival Another consideration for improving patient outcomes is first-line chemotherapy duration. While the ABC-02 study limited treatment to 8 cycles, a retrospective institutional database study showed that patients receiving $\geq 9$ cycles had significantly improved outcomes when compared with those who received $2-8$ cycles $(\mathrm{n}=382$; mPFS 13.3 versus 4.1 months, $\mathrm{P}<0.001$; mOS 22.1 versus 9.2 months, $\mathrm{P}<0.001)(80)$. These observations suggest the potential for continuation of first-line chemotherapy to improve survival. However, this study does not differentiate those patients receiving 8 cycles or less than 8 cycles from those receiving $\geq 9$ cycles, which could constitute significant bias.

\section{ABC-06 results for a modified FOLFOX regimen in second-line therapy}

First preliminary results from the ABC-06 randomized clinical trial, which compared modified FOLFOX $(\mathrm{mFOLFOX})+$ ASC with ASC alone in patients who had failed first-line GEM/CIS treatment ( $\mathrm{n}=162$, PS $0-1)$, showed a modest advantage of $\mathrm{mFOLFOX}+$ ASC versus ASC alone: mOS 6.2 versus 5.3 months, 6-month OS rate $50.6 \%$ versus $35.5 \%$, and 12 -month OS rate $25.9 \%$ versus 11.4\% (adjusted HR 0.69, CI: 0.50-0.97, $\mathrm{P}=0.031$ ) (23). The ABC-06 authors propose mFOLFOX as the new second-line standard of care treatment on the basis of a clinically meaningful increase in 6- and 12-month OS rates. However, given the similarity of these results to other second-line regimens, it could be argued that the most appropriate second-line regimen still remains to be defined.

\section{Etoposide-based regimens}

Although not commonly used, etoposide-based regimens were initially amongst the most promising options for the treatment of patients with BTC. For example, the etoposide/ $5 \mathrm{FU} /$ leucovorin $(\mathrm{LV})$ combination was the first treatment regimen to demonstrate a survival advantage over BSC: mOS 6.0 versus 2.5 months (21). Another study compared etoposide/5FU/LV (FELV) and epirubicin/CIS/5-FU (ECF) for the first-line treatment of BTC (81); unfortunately, targets for patient recruitment were not met due to the rarity of the disease, and it is likely that the small patient numbers 
underlie the observed statistically non-significant group differences ( $\mathrm{n}=54$, FELV: $\mathrm{mOS} 12.03$ months (CI: 9.3-14.7), 1 year OS 50.2\% (CI: 30.0-67.3) vs. ECF: mOS 9.02 months (CI: 6.46-11.51), $\mathrm{P}=0.2059$; 1 year OS 21\% (CI: 7.8-38.6). Furthermore, these results were achieved despite a known high prevalence of multi-drug resistance for etoposide in CCA (82-84) leading to further potential survival benefits if multi-drug resistance can be overcome. CAP7.1, a new drug that is converted to active etoposide by carboxylesterase-2 (CES-2), demonstrated a 1,000-fold higher cytotoxicity compared to etoposide $(85,86)$. In a Phase I trial conducted in 19 patients with advanced refractory solid tumors, median OS was 6.5 months (range, $2.5-25.63$ months) with a stage IV GBC patient experiencing an OS of 25.6 months and a CCA patient experiencing a PFS of 5.4 months (87). A subsequent randomized, BSC-controlled, Phase II second-line trial in 27 patients with crossover to etoposide toniribate permitted for BSC patients upon disease progression, showed favorable outcomes for etoposide toniribate versus BSC: DCR 55.6\% (CI: $21.2-86.3$ ) versus $20 \%$ (CI: $2.5-55.6), \mathrm{P}=0.13$; mPFS 3.4 versus 1.3 months, $\mathrm{P}=0.006$, and $\mathrm{mOS}$ for CAP7.1 7.5 months (CI: 1.7-15.8) (88). Estimated 1-year survival for patients receiving etoposide toniribate was $44 \%$ (88).

\section{First Phase II modified FOLFIRINOX study in BTC}

Further data has emerged from a Phase II study of FOLFIRINOX (irinotecan, leucovorin, fluorouracil and oxaliplatin) in second-line treatment of BTC ( $\mathrm{n}=30$, two-stage Simon's design) (89). The observed partial response rate was $10 \%$, DCR 67\%, mOS 10.7 months and PFS 6.2 months, with an acceptable safety profile. Modified FOLFIRINOX is currently under investigation as first-line treatment in the Phase II/III AMEBICA trial (NCT02591030).

\section{Targeted therapies, immunotherapy and beyond}

\section{Currently, targeted therapies show no to only marginal survival benefit}

A review by Sahu et al. reported no to marginal survival benefits for targeted therapies (monoclonal antibodies, tyrosine kinase inhibitors) mostly in combination with GEM-based regimens (mOS range 4.4-12.9 months; mPFS range 1.7-9.7 months), with variability likely due to undifferentiated patient populations (mixed molecular/ genetic profiles) (90). In a review by Chong and Zhu, mPFS was 1.6-8.8 months, mOS was 4.4-15.7 months (except for one study), with some marginal improvements in mOS of 13.5-15.7 months achieved with a variety of first-line combinations: GEM \pm platinum plus cetuximab (three trials), sorafenib (one trial), or cediranib (one trial) (90). Only one Phase II study of GEMOX + panitumumab in preselected Kirsten ras (KRAS) wild type patients reached a considerably longer mPFS of 10.6 months and mOS of 20.3 months (91). These figures compare to mPFS of 8.0 months and mOS of 11.7 months observed in ABC-02 (36).

In a third review, mPFS was $2-8$ months and mOS 4.4-20.0 months, mainly for GEM \pm platinum + targeted therapy trials (bevacizumab, sorafenib, vandetanib) (92). Finally, the benefits of targeted therapy in combination with GEM-based chemotherapy (mTTP 7.1 months, mOS 12.7 months), or GEM/platinum/FU (mTTP 9.0 months, mOS 12.5 months) over GEM/platinum (mTTP 5.3 months, mOS 9.5 months) have been demonstrated, suggesting EGFR-targeted therapy added to GEM-based regimens or GEM/platinum/FU as a new first-line standard for good PS patients (69).

\section{Many new genetic aberrations and thus potential targets for therapy have been identified}

Recently, potential new drug targets were discovered through identification of genetic aberrations in anatomical subtypes (Table 7) (90-92). Typically, however, a large patient percentage does not exhibit specific genetic aberrations, which may explain the observed limited effects of targeted therapy. Thus, a need exists for future studies to preselect patients through genetic profiling $(92,94)$.

\section{Four distinct molecular genetic clusters with different etiology, targets for potential therapy, and survival/ prognosis exist. Anatomic subtypes, however, do not differ in genetic background}

Four distinct genetic BTC clusters were recently identified through a whole-genome and epigenomic analysis of 489 BTC cases from 10 different countries (133 liver flukepositive and 356 fluke-negative cases) (Figure 4) (95). While Cluster 1 was mainly comprised of fluke-positive tumors, Cluster 2 had a fluke-positive/negative mix, and Clusters 3 and 4 were predominantly fluke-negative (95). These findings confirm genetically different molecular signatures indicating different carcinogenesis mechanisms for fluke-positive (extrinsic carcinogen) and -negative 
Table 7 New drug targets for genomic aberrations of biliary tract carcinoma anatomical subtypes and GBC

\begin{tabular}{|c|c|c|c|c|}
\hline $\begin{array}{l}\text { BTC } \\
\text { subtype/GBC }\end{array}$ & $\begin{array}{l}\text { Genetic aberration } \\
(91,92)^{\star}\end{array}$ & \multicolumn{3}{|r|}{ Molecular spectrum (93) } \\
\hline \multirow{3}{*}{ iBTC } & IDH1/2 mutations & $\mathrm{IDH} 1 / 2$ & $22-28 \%$ & AG-120, AG-881 \\
\hline & TP53* & - & $3-36 \% *$ & None reported \\
\hline & ARID1A* & - & $19-36 \% *$ & Histone deacetylase (HDAC) inhibitors, e.g., vorinostat and panobinostat \\
\hline \multirow[t]{3}{*}{ eBTC } & - & $\begin{array}{l}\text { HER2/neu } \\
\text { (mutation) }\end{array}$ & $11-20 \%$ & Tyrosine kinase inhibitors, e.g., afatinib, neratinib and dacomitinib \\
\hline & KRAS mutations ${ }^{*}$ & - & $40 \%{ }^{*}$ & None reported \\
\hline & TP53* $^{*}$ & - & $45 \% *$ & None reported \\
\hline pBTC & KRAS mutations & - & - & None reported \\
\hline \multirow[t]{3}{*}{ GBC } & EGFR & EGFR & $4-13 \%$ & Erlotinib, cetuximab \\
\hline & & $\begin{array}{l}\text { HER2/neu } \\
\text { (amplification) }\end{array}$ & $10-15 \%$ & Trastuzumab, lapatinib, pertuzumab, T-DM1 \\
\hline & ERBB2 and ERBB3 & ERBB3 & $0-12 \%$ & Seribantumab (MM-121), pertuzumab, trastuzumab, T-DM1 \\
\hline
\end{tabular}

ARID1A, AT-rich interactive domain-containing protein 1A; AKT (also known as protein kinase B); BAP1, BRCA1-Associated Protein 1; dBTC, distal biliary tract carcinoma; EGFR, epidermal growth factor; ERBB2, erb-b2 receptor tyrosine kinase 2; ERBB3, erb-b3 receptor tyrosine kinase 3; FGFR2, fibroblast growth factor receptor 2; GBC, gall bladder cancer; HER2, human epidermal growth factor; iBTC, intrahepatic biliary tract carcinoma; IDH1/2; isocitrate dehydrogenase 1 and 2; KRAS, Kirsten rat sarcoma viral oncogene homolog; mTOR, mammalian target of rapamycin; pBTC, perihilar biliary tract carcinoma; PI3K, phosphoinositide 3-kinase; PIK3CA, phosphoinositide-3-kinase catalytic alpha polypeptide; PRKACA/B; protein kinase CAMP-activated catalytic subunit alpha/beta; PTEN, phosphatase and tensin; T-DM1, trastuzumab emtansine; TP 53, tumor protein p53; TSC1, tuberous sclerosis protein 1.

(intrinsic genetic alteration) tumours (95). This study also disproved the theory of different anatomic BTC subtypes having distinct molecular-genetic backgrounds. Tumors at the same anatomical site exhibited profound molecular profile differences, while tumors at different anatomical sites displayed molecular profile similarities, thereby confirming that anatomical site is not a driver of molecular subtypes (95). However, the majority of Clusters $1 / 2$ were pCCA and dCCA, while Clusters $3 / 4$ were composed mostly of iCCA. While survival trends do not differ for anatomical subtypes $(32,37)$, for genetic clusters survival advantages are evident: Clusters 3/4 (mainly iCCA) demonstrated moderate significantly longer survival compared with Clusters 1/2 (mainly pCCA and dCCA, $\mathrm{P}<0.001$ (Figure 5) (95).

Molecular-genetic profiling, therefore, highlights distinct targeted therapy opportunities for each cluster with, e.g., Clusters $1 / 2$ potentially being susceptible to 


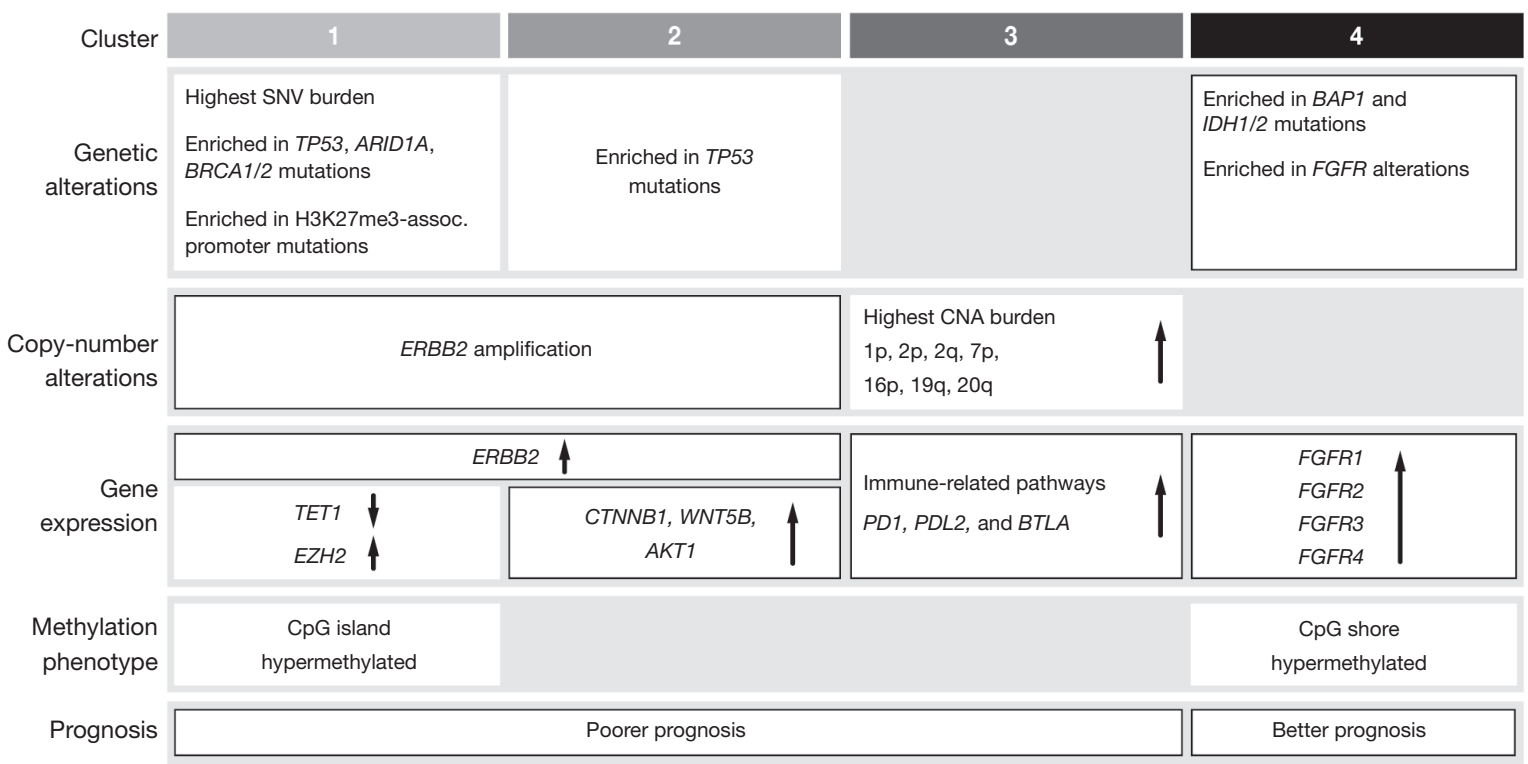

\begin{abstract}
AKT (also known as protein kinase B); ARID1A, AT-rich interactive domain-containing protein 1A; BAP1, BRCA1-Associated Protein 1; BRCA1/2, breast cancer genes 1/2; BTLA, B and T lymphocyte attenuator; CNA, copy number alteration; CNV, copy-number variants; CpG, cytosine base followed by a guanine base; CTNNB1, cadherin-associated protein beta 1; ERBB2, erb-b2 receptor tyrosine kinase 2; $\mathrm{EZH}$ 2, enhancer of zeste homolog 2; FGFR, fibroblast growth factor receptor; IDH1/2; isocitrate dehydrogenase 1 and 2; PD1, programmed cell death 1; PDL2; programmed cell death ligand 2; SNV, single-nucleotide variants; TET1, Tet Methylcytosine dioxygenase 1; TP53, tumor protein $\mathrm{p} 53$
\end{abstract}

Figure 4 Distinct genetic biliary tract carcinoma clusters (95).

human epidermal growth factor receptor 2 (ERBB2/HER2) targeting, Cluster 3 to immunotherapy (e.g., programmed cell death protein 1 (PD-1)-Inhibitors) and Cluster 4 to isocitrate dehydrogenase 1 (IDH) inhibitors or fibroblast growth factor receptor (FGFR)-targeting agents (90). These findings also highlight the potential benefit and limitations of novel PD-1-inhibitors $(96,97$ ), as only Cluster 3 (a small subgroup) seem susceptible.

Currently, there are numerous ongoing Phase II and III trials evaluating targeted therapies, particularly in second-line (98). Some include antiangiogenic therapy [e.g., apatinib targeting vascular endothelial growth factor receptor 2 (VEGFR2)], epidermal growth factor receptor (EGFR) and HER2 inhibitors (e.g., trastuzumab + chemotherapy), FGFR inhibitors (derazantinib, erdafitinib), IDH1 and tyrosine receptor kinase (TRK) inhibitors, and other inhibitors including olaparib and niraparib [targeting poly ADP ribose polymerase (PARP)]. Advanced BTC remains a challenge, and at present no targeted therapies have been validated for this disease $(93,98)$.

\section{Conclusions and future directions}

A high unmet need exists not only to develop effective therapies for BTC, but also to identify relevant prognostic factors and investigate their impact on treatment outcomes. Different treatment pathways exist within the collective patient journey, each associated with different survival outcomes, representable in a decision tree. As a patient moves through these decision points along their personal patient journey, the survival outcome changes even for the same treatment and patient; with the survival benefit of a particular treatment for a particular patient at a particular point in time depending not on the treatment itself, but also on the decision point and further individual patient/tumor specific prognostic factors.

In such a prognostic-factor driven model, mOS is a dynamic variable with an effect size range changing significantly around an overall median for a specific treatment at a particular decision point along the collective patient journey. Therefore, the classical approach of prescribing "one-chemotherapy-for-all-patients" on the 


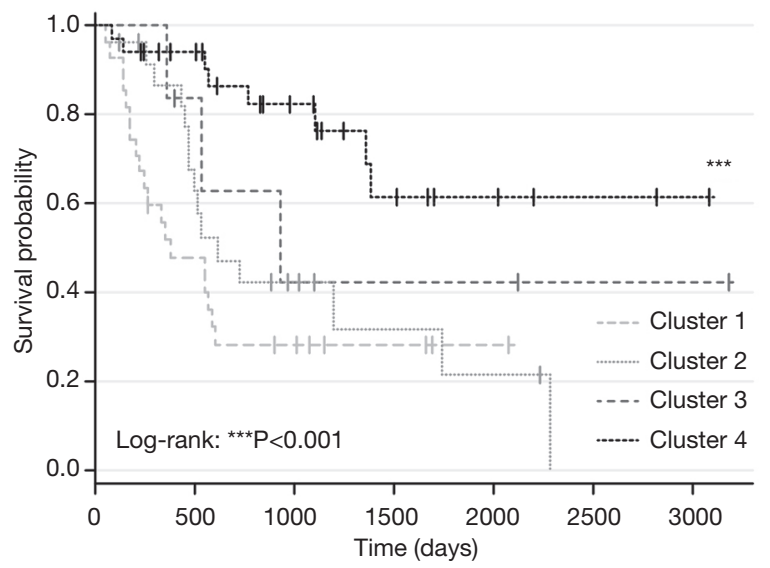

Figure 5 Differences in survival for the four different biliary tract carcinoma molecular clusters (95).

basis of results derived from summary statistics of one single mixed patient study may not be meaningful to an individual patient. For practical reasons many studies have enrolled resected and unresected patients and arrived at median survival figures for this patient mix. Clinically, however, these study results are not altogether meaningful at the individual patient level, as resected and unresected patients represent two distinct patient groups on completely different and no longer interchangeable treatment pathways within the treatment network. Furthermore, since resected patients generally have better outcomes, results are biased for both groups and therefore not directly transferable to clinical practice.

Thus, a paradigm shift is needed moving therapeutic decision-making more towards a personalized medicine approach. It is likely that future therapies will be developed for highly selected patient subgroups rather than for all CCA patients, while another part of future research will likely come from international, prospective studies in these subgroups. Future systemic therapy may be based on the combination of two separate treatment pillars, one "generalized-chemotherapeutic-pillar" as a treatment backbone for all patients (currently GEM/CIS in firstline), and one "individualized-targeted-therapy-pillar" utilizing targeted therapies, immunotherapies and/or other personalized medicines in highly pre-selected patient subgroups only. Thus, how original research is integrated and used by clinicians in a novel, dynamic and complex individualized therapeutic decision model is key to better treatment outcomes for this devastating disease.

\section{Acknowledgments}

Editorial support (in the form of writing assistance, collating author comments, assembling tables/figures, grammatical editing and referencing) was provided by Sarah Birch, PhD at Makara Health Communications Ltd, UK and was funded by Mundibiopharma Ltd.

Funding: This manuscript was funded by Mundibiopharma Ltd and CellAct GmbH.

\section{Footnote}

Conflicts of Interest: All authors have completed the ICMJE uniform disclosure form.(Available at http://dx.doi. org/10.21037/jgo-20-203). NU reports a leadership role, stock ownership, receipt of honoraria and research funding, and holding of patents at CellAct Pharma GmbH. The other authors have no conflicts of interest to declare.

Etbical Statement: The authors are accountable for all aspects of the work in ensuring that questions related to the accuracy or integrity of any part of the work are appropriately investigated and resolved.

Open Access Statement: This is an Open Access article distributed in accordance with the Creative Commons Attribution-NonCommercial-NoDerivs 4.0 International License (CC BY-NC-ND 4.0), which permits the noncommercial replication and distribution of the article with the strict proviso that no changes or edits are made and the original work is properly cited (including links to both the formal publication through the relevant DOI and the license). See: https://creativecommons.org/licenses/by-nc-nd/4.0/.

\section{References}

1. Valle JW, Borbath I, Khan SA, et al. Biliary cancer: ESMO Clinical Practice Guidelines for diagnosis, treatment and follow-up. Ann Oncol 2016;27:v28-v37.

2. Khuntikeo N, Chamadol N, Yongvanit P, et al. Cohort profile: cholangiocarcinoma screening and care program (CASCAP). BMC Cancer 2015;15:459.

3. Shaib Y, El-Serag HB. The epidemiology of cholangiocarcinoma. Semin Liver Dis 2004;24:115-25.

4. Bridgewater J, Galle PR, Khan SA, et al. Guidelines for the diagnosis and management of intrahepatic cholangiocarcinoma. J Hepatol 2014;60:1268-89.

5. Khan SA, Davidson BR, Goldin RD, et al. Guidelines for 
the diagnosis and treatment of cholangiocarcinoma: an update. Gut 2012;61:1657-69.

6. Ryerson AB, Eheman CR, Altekruse SF, et al. Annual Report to the Nation on the Status of Cancer, 1975-2012, featuring the increasing incidence of liver cancer. Cancer 2016;122:1312-37.

7. Yao KJ, Jabbour S, Parekh N, et al. Increasing mortality in the United States from cholangiocarcinoma: an analysis of the National Center for Health Statistics Database. BMC Gastroenterol 2016;16:117.

8. Razumilava N, Gores GJ. Cholangiocarcinoma. Lancet 2014;383:2168-79.

9. Rizvi S, Gores GJ. Pathogenesis, diagnosis, and management of cholangiocarcinoma. Gastroenterology 2013;145:1215-29.

10. Hoyos S, Navas MC, Restrepo JC, et al. Current controversies in cholangiocarcinoma. Biochimica et biophysica acta Molecular basis of disease 2018;1864:1461-7.

11. Radtke A, Konigsrainer A. Surgical therapy of cholangiocarcinoma. Visc Med 2016;32:422-6.

12. Song W, Miao DL, Chen L. Survival rates are higher in married patients with biliary tract cancer: a populationbased study. Oncotarget 2018;9:9531-9.

13. Dhanasekaran R, Hemming AW, Zendejas I, et al. Treatment outcomes and prognostic factors of intrahepatic cholangiocarcinoma. Oncol Rep 2013;29:1259-67.

14. Kim YS, Hwang IG, Park SE, et al. Role of adjuvant therapy after $\mathrm{R} 0$ resection for patients with distal cholangiocarcinoma. Cancer Chemother Pharmacol 2016;77:979-85.

15. Ji JH, Song HN, Kim RB, et al. Natural history of metastatic biliary tract cancer (BTC) patients with good performance status (PS) who were treated with only best supportive care (BSC). Jpn J Clin Oncol 2015;45:256-60.

16. Lamarca A, Benafif $S$, Ross $P$, et al. Cisplatin and gemcitabine in patients with advanced biliary tract cancer (ABC) and persistent jaundice despite optimal stenting: Effective intervention in patients with luminal disease. Eur J Cancer 2015;51:1694-703.

17. Yonemoto N, Furuse J, Okusaka T, et al. A multi-center retrospective analysis of survival benefits of chemotherapy for unresectable biliary tract cancer. Jpn J Clin Oncol 2007;37:843-51.

18. Sharma A, Dwary AD, Mohanti BK, et al. Best supportive care compared with chemotherapy for unresectable gall bladder cancer: a randomized controlled study. J Clin Oncol 2010;28:4581-6.
19. Dwary AD, Sharma A, Mohanti BK, et al. A randomized controlled trial (RCT) comparing best supportive care (BSC), 5-FU plus folinic acid (FUFA) and, gemcitabine plus oxaliplatin $(\mathrm{GemOx})$ in management of unresectable gallbladder cancer (GBC). J Clin Oncol 2009;27:4521.

20. Horgan A, Knox J, Aneja P, et al. Patterns of care and treatment outcomes in older patients with biliary tract cancer. Oncotarget 2015;6:44995-5004.

21. Glimelius B, Hoffman K, Sjoden PO, et al. Chemotherapy improves survival and quality of life in advanced pancreatic and biliary cancer. Ann Oncol 1996;7:593-600.

22. Takada T, Nimura Y, Katoh H, et al. Prospective randomized trial of 5-fluorouracil, doxorubicin, and mitomycin $\mathrm{C}$ for non-resectable pancreatic and biliary carcinoma: multicenter randomized trial. Hepatogastroenterology 1998;45:2020-6.

23. Lamarca A, Palmer DH, Wasan HS, et al. ABC-06 I A randomised phase III, multi-centre, open-label study of Active Symptom Control (ASC) alone or ASC with oxaliplatin/5-FUchemotherapy (ASC+mFOLFOX) for patients (pts) with locally advanced/metastatic biliary tract cancers (ABC) previously-treated with cisplatin/ gemcitabine (CisGem) chemotherapy. J Clin Oncol 2019;37:abstr 4003.

24. Park J, Kim MH, Kim KP, et al. Natural history and prognostic factors of advanced cholangiocarcinoma without surgery, chemotherapy, or radiotherapy: a largescale observational study. Gut Liver 2009;3:298-305.

25. Primrose JN, Fox R, Palmer DH, et al. Adjuvant capecitabine for biliary tract cancer: The BILCAP randomized study. J Clin Oncol 2017;35:4006.

26. Edeline J, Bonnetain F, Phelip JM, et al. Gemox versus surveillance following surgery of localized biliary tract cancer: Results of the PRODIGE 12-ACCORD 18 (UNICANCER GI) phase III trial. J Clin Oncol 2017;35:225.

27. Spolverato G, Kim Y, Alexandrescu S, et al. Management and outcomes of patients with recurrent intrahepatic cholangiocarcinoma following previous curative-intent surgical resection. Ann Surg Oncol 2016;23:235-43.

28. Neuhaus P, Jonas S, Settmacher U, et al. Surgical management of proximal bile duct cancer: extended right lobe resection increases resectability and radicality. Langenbecks Arch Surg 2003;388:194-200.

29. Bergquist JR, Groeschl RT, Ivanics T, et al. Mixed hepatocellular and cholangiocarcinoma: a rare tumor with a mix of parent phenotypic characteristics. HPB 2016;18:886-92. 
30. Skipworth JR, Keane MG, Pereira SP. Update on the management of cholangiocarcinoma. Dig Dis 2014;32:570-8.

31. Ruys AT, Busch OR, Rauws EA, et al. Prognostic impact of preoperative imaging parameters on resectability of hilar cholangiocarcinoma. HPB Surg 2013;2013:657309.

32. Waseem D, Tushar P. Intrahepatic, perihilar and distal cholangiocarcinoma: Management and outcomes. Ann Hepatol 2017;16:133-9.

33. Hu HJ, Mao H, Shrestha A, et al. Prognostic factors and long-term outcomes of hilar cholangiocarcinoma: A singleinstitution experience in China. World J Gastroenterol 2016;22:2601-10.

34. Okusaka T, Nakachi K, Fukutomi A, et al. Gemcitabine alone or in combination with cisplatin in patients with biliary tract cancer: a comparative multicentre study in Japan. Br J Cancer 2010;103:469-74.

35. Primrose JN, Fox RP, Palmer DH, et al. Capecitabine compared with observation in resected biliary tract cancer (BILCAP): a randomised, controlled, multicentre, phase 3 study. Lancet Oncol 2019;20:663-73.

36. Valle J, Wasan H, Palmer DH, et al. Cisplatin plus gemcitabine versus gemcitabine for biliary tract cancer. $\mathrm{N}$ Engl J Med 2010;362:1273-81.

37. Ercolani G, Dazzi A, Giovinazzo F, et al. Intrahepatic, peri-hilar and distal cholangiocarcinoma: Three different locations of the same tumor or three different tumors? Eur J Surg Oncol 2015;41:1162-9.

38. Yoh T, Hatano E, Nishio T, et al. Significant improvement in outcomes of patients with intrahepatic cholangiocarcinoma after surgery. World J Surg 2016;40:2229-36.

39. Hundal R, Shaffer EA. Gallbladder cancer: epidemiology and outcome. Clin Epidemiol 2014;6:99-109.

40. Spolverato G, Bagante F, Ethun CG, et al. Defining the chance of statistical cure among patients with extrahepatic biliary tract cancer. World J Surg 2017;41:224-31.

41. Yamamoto M, Ariizumi S. Surgical outcomes of intrahepatic cholangiocarcinoma. Surg Today 2011;41:896-902.

42. Mavros MN, Economopoulos KP, Alexiou VG, et al. Treatment and prognosis for patients with intrahepatic cholangiocarcinoma: systematic review and meta-analysis. JAMA Surg 2014;149:565-74.

43. Lubezky N, Facciuto M, Harimoto N, et al. Surgical treatment of intrahepatic cholangiocarcinoma in the USA. J Hepatobiliary Pancreat Sci 2015;22:124-30.

44. Bhardwaj N, Garcea G, Dennison AR, et al. The surgical management of Klatskin tumours: has anything changed in the last decade? World J Surg 2015;39:2748-56.

45. Wellner UF, Shen Y, Keck T, et al. The survival outcome and prognostic factors for distal cholangiocarcinoma following surgical resection: a meta-analysis for the 5-year survival. Surg Today 2017;47:271-9.

46. Brunner TB, Seufferlein T. Radiation therapy in cholangiocellular carcinomas. Best Pract Res Clin Gastroenterol 2016;30:593-602.

47. Mizuno T, Ebata T, Yokoyama Y, et al. Adjuvant gemcitabine monotherapy for resectable perihilar cholangiocarcinoma with lymph node involvement: a propensity score matching analysis. Surg Today 2017;47:182-92.

48. Doherty MK, Knox JJ. Adjuvant therapy for resected biliary tract cancer: a review. Chin Clin Oncol 2016;5:64.

49. Horgan AM, Amir E, Walter T, et al. Adjuvant therapy in the treatment of biliary tract cancer: a systematic review and meta-analysis. J Clin Oncol 2012;30:1934-40.

50. Schweitzer N, Weber T, Kirstein MM, et al. The effect of adjuvant chemotherapy in patients with intrahepatic cholangiocarcinoma: a matched pair analysis. J Cancer Res Clin Oncol 2017;143:1347-55.

51. Groot Koerkamp B, Wiggers JK, Allen PJ, et al. Recurrence rate and pattern of perihilar cholangiocarcinoma after curative intent resection. J Am Coll Surg 2015;221:1041-9.

52. Komaya K, Ebata T, Shirai K, et al. Recurrence after resection with curative intent for distal cholangiocarcinoma. Br J Surg 2017;104:426-33.

53. Edeline J, Benabdelghani M, Bertaut A, et al. Gemcitabine and oxaliplatin chemotherapy or surveillance in resected biliary tract cancer (PRODIGE 12-ACCORD 18-UNICANCER GI): a randomized Phase III study. J Clin Oncol 2019;37:658-67.

54. Doussot A, Groot-Koerkamp B, Wiggers JK, et al. Outcomes after Resection of Intrahepatic Cholangiocarcinoma: External Validation and Comparison of Prognostic Models. J Am Coll Surg 2015;221:452-61.

55. Reames BN, Ejaz A, Koerkamp BG, et al. Impact of major vascular resection on outcomes and survival in patients with intrahepatic cholangiocarcinoma: A multiinstitutional analysis. J Surg Oncol 2017;116:133-9.

56. Miyazaki Y, Kokudo T, Amikura K, et al. Survival of surgery for recurrent biliary tract cancer: a single-center experience and systematic review of literature. Jpn J Clin Oncol 2017;47:206-12.

57. Luvira V, Eurboonyanun C, Bhudhisawasdi V, et al. 
Patterns of recurrence after resection of mass-forming type intrahepatic cholangiocarcinomas. Asian Pac J Cancer Prev 2016;17:4735-9.

58. Eckel F, Schmid RM. Chemotherapy in advanced biliary tract carcinoma: a pooled analysis of clinical trials. Br J Cancer 2007;96:896-902.

59. Agarwal R, Sendilnathan A, Siddiqi NI, et al. Advanced biliary tract cancer: clinical outcomes with ABC-02 regimen and analysis of prognostic factors in a tertiary care center in the United States. J Gastrointest Oncol 2016;7:996-1003.

60. Valle JW, Furuse J, Jitlal M, et al. Cisplatin and gemcitabine for advanced biliary tract cancer: a meta-analysis of two randomised trials. Ann Oncol 2014;25:391-8.

61. Kameda R, Ando T, Kobayashi S, et al. A retrospective study of chemotherapy with cisplatin plus gemcitabine after the failure of gemcitabine monotherapy for biliary tract cancer. Jpn J Clin Oncol 2013;43:636-40.

62. Yamashita Y, Sirabe K, Kawabe K, et al. Gemcitabine in Combination with Cisplatin in Patients with Unresectable Advanced or Recurrent Biliary Tract Cancer--A Multicenter Prospective Observational Study in Fukuoka. Gan To Kagaku Ryoho 2015;42:1185-9.

63. Fiteni F, Nguyen T, Vernerey D, et al. Cisplatin/ gemcitabine or oxaliplatin/gemcitabine in the treatment of advanced biliary tract cancer: a systematic review. Cancer Med 2014;3:1502-11.

64. Valle JW, Wasan H, Jitlal M, et al. ABC-03: A randomized phase II trial of cediranib (AZD2171) or placebo in combination with cisplatin/gemcitabine (CisGem) chemotherapy for patients (pts) with advanced biliary tract cancer (ABC). J Clin Oncol 2014;32:4002.

65. Woo SM, Lee WJ, Kim JH, et al. Gemcitabine plus cisplatin versus capecitabine plus cisplatin as firstline chemotherapy for advanced biliary tract cancer: a retrospective cohort study. Chemotherapy 2013;59:232-8.

66. Cho H, Kim B, Yoo C, et al. Prognostic factors in patients (pts) with advanced biliary tract cancer (BTC) treated with first-line gemcitabine plus cisplatin (GEMCIS): retrospective analysis of 740 pts. J Clin Oncol 2017;2017:424.

67. Park JO, Oh DY, Hsu C, et al. Gemcitabine plus cisplatin for advanced biliary tract cancer: a systematic review. Cancer Res Treat 2015;47:343-61.

68. Ulahannan SV, Rahma OE, Duffy AG, et al. Identification of active chemotherapy regimens in advanced biliary tract carcinoma: a review of chemotherapy trials in the past two decades. Hepat Oncol 2015;2:39-50.

69. Eckel F, Schmid RM. Chemotherapy and targeted therapy in advanced biliary tract carcinoma: a pooled analysis of clinical trials. Chemotherapy 2014;60:13-23.

70. Valle JW. Advances in the treatment of metastatic or unresectable biliary tract cancer. Ann Oncol 2010;21 Suppl 7:vii345-vii348.

71. Lamarca A, Ross P, Wasan HS, et al. Advanced Intrahepatic Cholangiocarcinoma: Post Hoc Analysis of the ABC-01, -02, and -03 Clinical Trials. J Natl Cancer Inst 2020;112:200-10.

72. Brieau B, Dahan L, De Rycke Y, et al. Second-line chemotherapy for advanced biliary tract cancer after failure of the gemcitabine-platinum combination: A large multicenter study by the Association des GastroEnterologues Oncologues. Cancer 2015;121:3290-7.

73. Fornaro L, Vivaldi C, Cereda S, et al. Second-line chemotherapy in advanced biliary cancer progressed to first-line platinum-gemcitabine combination: a multicenter survey and pooled analysis with published data. J Exp Clin Cancer Res 2015;34:156.

74. Jordan E, Abou-Alfa GK, Lowery MA. Systemic therapy for biliary cancers. Chin Clin Oncol 2016;5:65.

75. Jung JH, Lee HS, Jo JH, et al. Combination Therapy with Capecitabine and Cisplatin as Second-Line Chemotherapy for Advanced Biliary Tract Cancer. Chemotherapy 2017;62:361-6.

76. Takahara N, Nakai Y, Isayama H, et al. Second-line chemotherapy in patients with advanced or recurrent biliary tract cancer: a single center, retrospective analysis of 294 cases. Invest New Drugs 2018;36:1093-102.

77. Kim ST, Oh SY, Lee J, et al. Capecitabine plus Oxaliplatin as a Second-Line Therapy for Advanced Biliary Tract Cancers: A Multicenter, Open-Label, Phase II Trial. J Cancer 2019;10:6185-90.

78. Lamarca A, Hubner RA, David Ryder W, et al. Secondline chemotherapy in advanced biliary cancer: a systematic review. Ann Oncol 2014;25:2328-38.

79. Ying J, Chen J. Combination versus mono-therapy as salvage treatment for advanced biliary tract cancer: A comprehensive meta-analysis of published data. Crit Rev Oncol Hematol 2019;139:134-42.

80. Doherty MK, McNamara MG, Aneja P, et al. Long term responders to palliative chemotherapy for advanced biliary tract cancer. J Gastrointest Oncol 2017;8:352-60.

81. Rao S, Cunningham D, Hawkins RE, et al. Phase III study of 5FU, etoposide and leucovorin (FELV) compared to epirubicin, cisplatin and 5FU (ECF) in previously 
untreated patients with advanced biliary cancer. $\mathrm{Br} \mathrm{J}$

Cancer 2005;92:1650-4.

82. Cao L, Duchrow M, Windhovel U, et al. Expression of MDR1 mRNA and encoding P-glycoprotein in archival formalin-fixed paraffin-embedded gall bladder cancer tissues. Eur J Cancer 1998;34:1612-7.

83. Marin JJG, Lozano E, Herraez E, et al. Chemoresistance and chemosensitization in cholangiocarcinoma.

Biochimica et biophysica acta Molecular basis of disease 2018;1864:1444-53.

84. Tepsiri N, Chaturat L, Sripa B, et al. Drug sensitivity and drug resistance profiles of human intrahepatic cholangiocarcinoma cell lines. World J Gastroenterol 2005;11:2748-53.

85. Schroeder U, Bernt KM, Lange B, et al. Hydrolytically activated etoposide prodrugs inhibit MDR-1 function and eradicate established MDR-1 multidrug-resistant T-cell leukemia. Blood 2003;102:246-53.

86. Wrasidlo W, Schroder U, Bernt K, et al. Synthesis, hydrolytic activation and cytotoxicity of etoposide prodrugs. Bioorg Med Chem Lett 2002;12:557-60.

87. Keilholz U, Rohde L, Mehlitz P, et al. First-in-man dose escalation and pharmacokinetic study of CAP7.1, a novel prodrug of etoposide, in adults with refractory solid tumours. Eur J Cancer 2017;80:14-25.

88. Pape UF, Kasper S, Meiler J, et al. Randomized Phase II trial of the carboxylesterase (CES)-converted novel drug EDO-S7.1 in patients (pts) with advanced biliary tract cancers (BTC). J Clin Oncol 2019;37:abstr 264.

89. Belkouz A, de Voss-Geelen J, Eskens F, et al. Efficacy and safety of FOLFIRINOX in advanced biliary tract cancer

Cite this article as: Jansen H, Pape UF, Utku N. A review of systemic therapy in biliary tract carcinoma. J Gastrointest Oncol 2020;11(4):770-789. doi: 10.21037/jgo-20-203 after failure of gemcitabine plus cisplatin: A phase II trial. J Clin Oncol 2019;37.

90. Sahu S, Sun W. Targeted therapy in biliary tract cancerscurrent limitations and potentials in the future. $\mathrm{J}$ Gastrointest Oncol 2017;8:324-36.

91. Chong DQ, Zhu AX. The landscape of targeted therapies for cholangiocarcinoma: current status and emerging targets. Oncotarget 2016;7:46750-67.

92. Simone V, Brunetti O, Lupo L, et al. Targeting angiogenesis in biliary tract cancers: an open option. Int J Mol Sci 2017;18:418.

93. Jain A, Javle M. Molecular profiling of biliary tract cancer: a target rich disease. J Gastrointest Oncol 2016;7:797-803.

94. Ghidini M, Pizzo C, Botticelli A, et al. Biliary tract cancer: current challenges and future prospects. Cancer Manag Res 2018;11:379-88.

95. Jusakul A, Cutcutache I, Yong CH, et al. Wholegenome and epigenomic landscapes of etiologically distinct subtypes of cholangiocarcinoma. Cancer Discov 2017;7:1116-35.

96. Khoja L, Butler MO, Kang SP, et al. Pembrolizumab. J Immunother Cancer 2015;3:36.

97. Sul J, Blumenthal GM, Jiang X, et al. FDA Approval Summary: Pembrolizumab for the treatment of patients with metastatic non-small cell lung cancer whose tumors express Programmed Death-Ligand 1. Oncologist 2016;21:643-50.

98. Vienot A, Neuzillet C. Cholangiocarcinoma: the quest for a second-line systemic treatment. Transl Cancer Res 2018:S275-S288. 